\title{
Gradiente litorâneo-limnético do fitoplâncton e ficoperifíton em uma lagoa da planície de inundação do Rio Mogi-Guaçu
}

\author{
GLÓRIA M. TANIGUCHI ${ }^{1,4}$, DENISE C. BICUDO ${ }^{2}$ e PEDRO A.C. SENNA ${ }^{\dagger 3}$
}

(recebido: 29 de janeirode 2004; aceito: 11 de novembro de 2004)

\begin{abstract}
Phytoplankton and phycoperiphyton littoral-limnetic gradient of in an oxbow lake of Mogi-Guaçu River floodplain). Study aimed at evaluating the phytoplankton and phycoperiphyton structure and dynamics during a hydrological cycle at the littoral-limnetic gradient in the Diogo Pond, and oxbow lake of the Mogi-Guaçu river floodplain (São Paulo State, Brazil). Samplings were carried out during one hydrological cycle (high water, flooding, low water, and drought) in three sites (open water, littoral-limnetic interface, and within the macrophyte stand) for phytoplankton, and in the littoral region for phycoperiphyton associated to Eicchornia azurea Kunth. Algal classes densities and biomass (chlorophyll-a) were analyzed for both communities. Principal component analysis separated the two major hydrological periods: the rainy and the dry seasons. Rainy season was associated to higher temperatures, higher water levels, lower nitrate, and higher densities of Euglenophyceae, Cryptophyceae, Chrysophyceae, and chlorophyll- $a$ concentrations. Disturbance regime during high water and flood periods favoured opportunistic groups represented by phytoflagellates. Moreover, phytoplankton from littoral region was separated during high water, low water and drought phases indicating the influence of the spatial gradient. Periphyton showed a distinct trend, with higher biomass and densities during low water and drought phases. Hydrological cycle was considered the main driving force on the phytoplankton and periphyton structure as well as on the interaction and interchanges of these communities in the oxbow pond.
\end{abstract}

Key words - floodplain, litoral-limnetic gradient, oxbow lake, periphyton, phytoplankton

RESUMO - (Gradiente litorâneo-limnético do fitoplâncton e ficoperifíton em uma lagoa da planície de inundação do Rio MogiGuaçu). O estudo visou analisar a estrutura e dinâmica do fitoplâncton e ficoperifíton durante um ciclo hidrológico no gradiente litorâneo-limnético na Lagoa do Diogo, uma lagoa marginal da planície de inundação do Rio Mogi-Guaçu (Estado de São Paulo). Foram realizadas quatro coletas durante o ciclo hidrológico (enchente, cheia, vazante e seca) em três locais (água aberta, interface limnético-litorânea e dentro do banco de macrófitas aquáticas) para o fitoplâncton e na região litoral para o perifíton associado a Eichhornia azurea Kunth. Biomassa (clorofila- $a$ ) e densidade das classes de algas foram analisadas para ambas comunidades. Análise de componentes principais separou as duas maiores fases hidrológicas: chuvosa e seca. A primeira foi associada às maiores temperaturas, nível de água, menores valores de nitrato e às maiores densidades de Euglenophyceae, Cryptophyceae e Chrysophyceae e aos maiores teores de clorofila- $a$. O maior regime de perturbações nas épocas de enchente e cheia favoreceu grupos oportunistas, representados pelos fitoflagelados. Houve, ainda, a separação do fitoplâncton da região litoral nas épocas de cheia, vazante e seca, indicando a influência do gradiente espacial do sistema. A comunidade perifítica apresentou tendência distinta da fitoplanctônica, com maiores valores de biomassa e densidades durante os períodos de vazante e seca. O regime hidrológico foi considerado a principal função de força sobre a estrutura do fitoplâncton e perifíton, bem como sobre a interação e o intercâmbio de algas entre essas comunidades na lagoa marginal.

Palavras-chave - fitoplâncton, gradiente litorâneo-limnético, lagoa marginal, planície de inundação, perifíton

\section{Introdução}

As lagoas de planícies de inundação, pelo fato de constituírem ambientes lênticos durante a maior parte do ciclo hidrológico, apresentam condições propícias

\footnotetext{
1. Empresa Brasileira de Pesquisa Agropecuária, Centro Nacional de Pesquisa do Algodão, Rua Osvaldo Cruz, 1143, 58107-720 Campina Grande, PB, Brasil.

2. Instituto de Botânica, Seção de Ecologia, Caixa Postal 4005 , 01061-970 São Paulo, SP, Brasil

3. Universidade Federal de São Carlos, Departamento de Ecologia e Biologia Evolutiva, Caixa Postal 676, 13565-905 São Carlos, SP, Brasil.

4. Autor para correspondência: gloria@cnpa.embrapa.br
}

para o desenvolvimento de comunidades fitoplanctônicas, perifíticas e de macrófitas aquáticas, o que se reflete nas altas taxas de produtividade primária destes ecossistemas (Bonetto et al. 1984). Apesar da grande abundância de áreas alagáveis em escala mundial, Goldsborough \& Robinson (1996) consideram que a pesquisa ficológica tem se concentrado em outros sistemas como rios e lagos, de forma que informações sobre as comunidades algais nos ambientes alagáveis são, de modo geral, bastante fragmentadas, particularmente para as áreas tropicais e subárticas.

No Brasil, já foram publicados vários trabalhos relacionados à comunidade fitoplanctônica, voltados para aspectos taxonômicos e ecológicos (Barbosa et al. 
1995). Porém, estudos sobre comunidades ficoperifíticas são bem menos numerosos e só se tornaram mais expressivos a partir da década de 1980 (Bicudo et al. 1995). Apesar da grande representatividade de áreas alagáveis no Brasil e, assim, de regiões de interface terra-água, o conhecimento sobre as comunidades de algas nesse gradiente espacial é, ainda, muito escasso.

Para a Estação Ecológica de Jataí, os estudos relacionados ao fitoplâncton e ao ficoperifíton foram realizados nas Lagoas do Infernão e Diogo, abrangendo aspectos como taxonomia, diversidade biológica, processos de nitrificação, efeitos do regime de inundação sobre a estrutura e a produção de comunidades, variações sazonais e espaciais, entre outros (Santos \& Pires 2000, Schwarzbold 1992, Schwarzbold et al. 1990, Magrin 1998, Magrin \& Senna 1997, Senna et al. 1998, Taniguchi et al. 1998, 2003). Esses trabalhos enfatizam principalmente a influência dos pulsos de inundação sobre as comunidades e os processo ocorrentes nas lagoas.

Até o momento não há publicações no Brasil sobre a dinâmica das comunidades de algas planctônicas e perifíticas no gradiente litorâneo-limnético. Apenas o trabalho de Taniguchi et al. (2000) traz informações sobre as comunidades algais no gradiente litorâneolimnético da Lagoa do Diogo, com a avaliação do intercâmbio populacional de desmídias planctônicas e perifíticas nesse gradiente.

O presente trabalho objetiva avaliar a dinâmica da estrutura de comunidades fitoplanctônicas e ficoperifíticas no gradiente litorâneo-limnético, bem como analisar a influência do ciclo hidrológico sobre essas comunidades. Pretende contribuir com o melhor entendimento das flutuações espacial e temporal das comunidades de algas em lagoas marginais de planícies de inundação tropicais.

\section{Material e métodos}

A Lagoa do Diogo, uma lagoa marginal da planície de inundação do Rio Mogi-Guaçu, está situada na unidade de conservação ambiental Estação Ecológica de Jataí "Conde Joaquim Augusto Ribeiro do Vale" (21 $1^{\circ} 33^{\prime}-21^{\circ} 37^{\prime} \mathrm{S}$ e $47^{\circ} 45^{\prime}$ $47^{\circ} 51^{\prime} \mathrm{W}$ ), região centro-leste do Estado de São Paulo (figura 1). De acordo com Pires et al. (2000), o clima é classificado como Aw, segundo o sistema de Köppen, com as temperaturas mais elevadas ocorrendo no período de maior precipitação (dezembro a fevereiro) e as menores no período de menor precipitação (maio a outubro). A lagoa é um sistema de drenagem com área de aproximadamente 4.452 ha. Possui uma conexão direta com o canal principal do rio durante o ano todo, além de receber influxo permanente do Córrego do

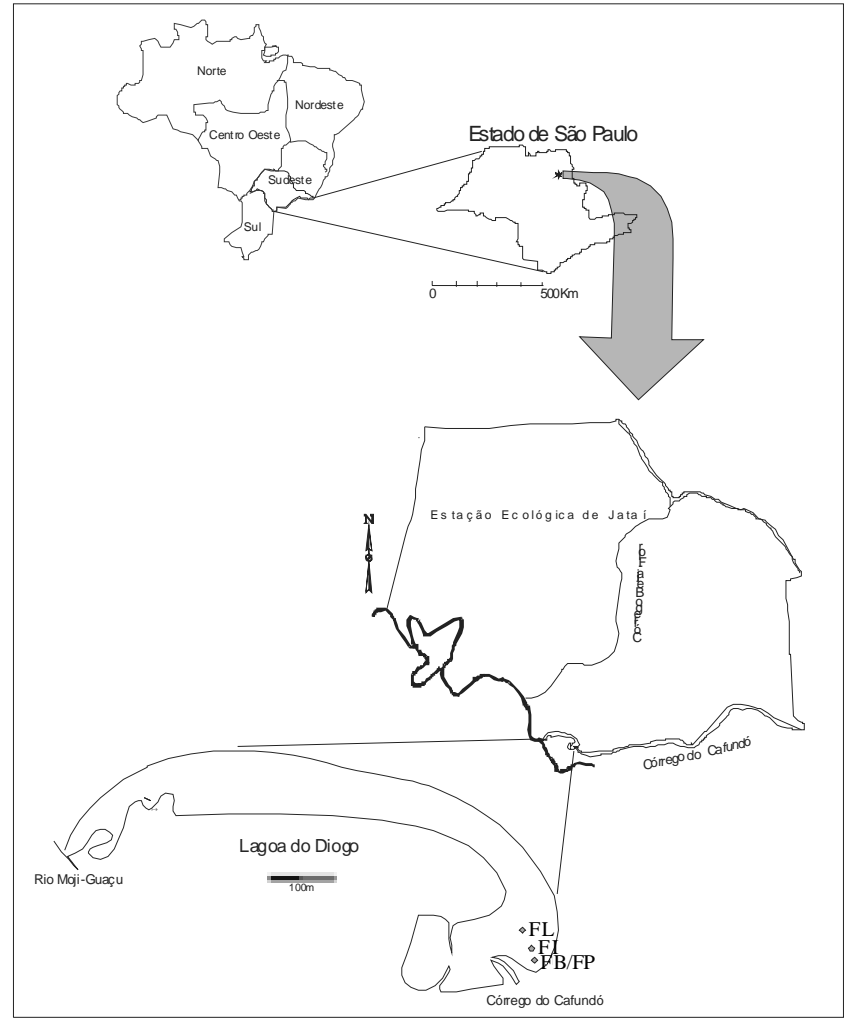

Figura 1. Localização da Lagoa do Diogo na Estação Ecológica de Jataí e das estações de amostragem: FL, FI e FB/FP.

Figure 1. Location of "Diogo" Pond on the Jataí Ecological Station and sampling sites: FL, FI, and FB/FP.

Cafundó em sua porção leste. As características morfométricas da lagoa são fornecidas por Krusche (1989).

Para coleta de material foram escolhidos quatro hábitats, localizados em três estações de amostragem, na extremidade próxima à desembocadura do córrego do Cafundó (figura 1):

FL: fitoplâncton limnético;

FI: fitoplâncton da região de interface litoral-limnética (limite entre banco de Eichhornia azurea e água aberta);

FB: fitoplâncton dentro do banco de E. azurea (da região litoral);

FP: ficoperifíton associado a E. azurea.

Foram realizadas coletas em quatro períodos de forma a englobar um ciclo hidrológico: enchente (28/11/1996), cheia (18/02/1997), vazante (15/04/1997) e seca (18/08/1997). Foram totalizadas 12 amostras de material ficoperifítico (FP) (quatro para análise qualitativa e oito para análise quantitativa, incluindo réplicas) e 36 amostras de material fitoplanctônico (FL, FI e FB ) (12 para a análise qualitativa e 24 para a análise quantitativa, incluindo réplicas), além de 32 amostras para clorofila- $a$ (24 de material planctônico e oito de material perifítico, incluindo réplicas).

Para a análise qualitativa, o perifíton foi amostrado através de procedimento randômico, isto é, por sorteio de pecíolos de E. azurea. Pedaços de pecíolo de, 
aproximadamente, $7 \mathrm{~cm}$ foram cortados e transportados até o laboratório. Em laboratório, o material ficoperifítico foi removido do substrato por meio de raspagem com lâminas de barbear e jatos d'água destilada. O material fitoplanctônico foi coletado na subsuperfície da água com rede de plâncton de $20 \mu \mathrm{m}$ de abertura de malha. O material coletado foi acondicionado em frascos de polietileno e fixado em formol 4\%. Para a classificação sistemática das algas, adotou-se o sistema de Round (1965, 1971).

Para análise quantitativa, o método de coleta e remoção do ficoperifíton foi o mesmo da análise qualitativa, porém a área do substrato foi determinada. O fitoplâncton foi coletado diretamente da subsuperfície da água com frascos de polietileno. As amostras coletadas foram preservadas em lugol acético a $0,5 \%$. A quantificação seguiu o método descrito por Utermöhl (1958) e o tempo de sedimentação foi de três horas para cada centímetro de altura da câmara (Margalef 1983). O limite de contagem foi estabelecido pelo método da curva de rarefação de espécies conforme Bicudo (1990). Os cálculos da densidade do fitoplâncton e do ficoperifíton (adaptados para a área do substrato) foram realizados de acordo com as fórmulas apresentadas por Ros (1979).

A técnica utilizada para a análise da clorofila- $a$, corrigida para feofitina, foi a de Nusch \& Palme (1975) e os cálculos foram realizados de acordo com o método descrito por Wetzel \& Likens (1991).

Os métodos e equipamentos usados para análise das variáveis físicas e químicas da água constam na tabela 1.

Para a ordenação dos períodos hidrológicos e locais de amostragem foi aplicada a análise multivariada de componentes principais - ACP (matriz de correlação), seguindo o método de Kaiser (Manly 1986), com auxílio do programa "Statistica For Windows - Versão 5.5", licenciado para a
Universidade Federal de São Carlos. A ACP foi dividida em duas fases: (1) utilizando apenas os dados biológicos como descritores (clorofila- $a$, densidade total e classes fitoplanctônicas) e (2) utilizando dados das variáveis físicas, químicas e biológicas como descritores (clorofila- $a$, densidade fitoplanctônica total e dos grupos algais de maior importância, obtido pela análise anterior). Nessas análises não foram incluídos os dados referentes ao ficoperifíton, devido às diferenças de unidades (expressas em área e não em volume).

\section{Resultados}

Os valores médios, máximos, mínimos e erro padrão das variáveis físicas e químicas da Lagoa do Diogo obtidos no período em estudo constam na tabela 2.

A biomassa fotossintética (clorofila- $a$ ) e a densidade total da comunidade fitoplanctônica foram mais elevadas nas épocas de enchente e cheia e as menores concentrações foram registradas na vazante e seca (tabela 3). Todavia, distribuição mais eqüitativa entre as classes de algas foi encontrada nos dois últimos períodos, principalmente para as regiões de interface e litorânea. As classes mais bem representadas foram Cryptophyceae, Euglenophyceae e Chlorophyceae (figuras 2, 3). Os demais grupos (Bacillariophyceae, Cyanophyceae, Chloromonadophyceae, Dinophyceae, Xanthophyceae, Oedogoniophyceae e Zygnemaphyceae) ocorreram em baixas densidades e porcentagens no plâncton.

A análise de componentes principais (ACP), com base no fitoplâncton, resumiu $70 \%$ da variabilidade

Tabela 1. Métodos e equipamentos usados para as medidas das variáveis abióticas na Lagoa do Diogo.

Table 1. Methods and equipment used for abiotic variables measurements at "Diogo" Pond.

\begin{tabular}{ll}
\hline Variável & Método e/ou equipamento \\
\hline Transparência da Água $(\mathrm{m})$ & Disco de Secchi \\
Temperatura $\left({ }^{\circ} \mathrm{C}\right)$ & Horiba, modelo U-10 \\
$\mathrm{pH}$ & Horiba, modelo U-10 \\
Condutividade $\left(\mu \mathrm{S} . \mathrm{cm}^{-1}\right)$ & Horiba, modelo U-10 \\
Oxigênio Dissolvido $\left(\mathrm{mg} . \mathrm{L}^{-1}\right)$ & Método de Winkler modificado por Golterman et al. $(1978)$ \\
Nitrito $\left(\mu \mathrm{g} . \mathrm{L}^{-1}\right)$ & Filtros Whatman GF/F, Mackereth et al. $(1978)$ \\
Nitrato $\left(\mu \mathrm{g} . \mathrm{L}^{-1}\right)$ & Filtros Whatman GF/F, Mackereth et al. $(1978)$ \\
Amônio $\left(\mu \mathrm{g} . \mathrm{L}^{-1}\right)$ & Filtros Whatman GF/F, Koroleff $(1976)$ \\
Nitrogênio Total $\left(\mu \mathrm{g} . \mathrm{L}^{-1}\right)$ & Valderrama $(1981)$ \\
Ortofosfato $\left(\mu \mathrm{g} . \mathrm{L}^{-1}\right)$ & Filtros Whatman GF/F, Strickland \& Parsons $(1960)$ \\
Fósforo Total Dissolvido $\left(\mu \mathrm{g} . \mathrm{L}^{-1}\right)$ & Filtros Whatman GF/F, Strickland \& Parsons $(1960)$ \\
Fósforo Total $\left(\mu \mathrm{g} . \mathrm{L}^{-1}\right)$ & Valderrama $(1981)$ \\
Ortossilicato $\left(\mathrm{mg} . \mathrm{L}^{-1}\right)$ & Filtros Whatman GF/F, Golterman et al. (1978) \\
Matéria em Suspensão $\left(\mathrm{mg} . \mathrm{L}^{-1}\right)$ & Filtros Whatman GF/F, Teixeira et al. $(1965)$ \\
\hline
\end{tabular}


Tabela 2. Valores mínimos, médios e máximos e erro padrão das variáveis físicas e químicas observados durante as quatro coletas realizadas na Lagoa do Diogo. OD = oxigênio dissolvido, $\mathrm{NO}_{3}=$ nitrato, $\mathrm{NH}_{4}=$ amônio, $\mathrm{NT}=$ nitrogênio total, PTD = fósforo total dissolvido, PT = fósforo total, $\mathrm{SiH}_{4} \mathrm{O}_{4}=$ ortossilicato, $\mathrm{MIS}=$ material inorgânico em suspensão, MOS = material orgânico em suspensão, $\mathrm{NT} / \mathrm{PT}=$ razão atômica nitrogênio total e fósforo total.

Table 2. Minimum, medium, maximum, and standard error of physical and chemical variables observed during four field trips at Diogo Pond. $\mathrm{OD}=$ dissolved oxygen, $\mathrm{NO}_{3}=$ nitrate, $\mathrm{NH}_{4}=$ ammonium, $\mathrm{NT}=$ total nitrogen, $\mathrm{PTD}=$ total dissolved phosphorus, $\mathrm{PT}=$ total phosphorus, $\mathrm{SiH}_{4} \mathrm{O}_{4}=$ orthosilicate, MIS = inorganic suspended matter, $\mathrm{MOS}=$ organic suspended matter, NT/PT = total nitrogen and total phosphorus atomic ratio.

\begin{tabular}{|c|c|c|c|c|}
\hline \multirow[t]{2}{*}{ Variável } & \multicolumn{3}{|c|}{ Valor } & \multirow{2}{*}{$\begin{array}{c}\text { Erro } \\
\text { padrão }\end{array}$} \\
\hline & mínimo & médio & máximo & \\
\hline Profundidade (m) & 0,30 & 1,68 & 3,20 & 0,26 \\
\hline Tansparência (m) & 0,25 & 0,49 & 0,80 & 0,06 \\
\hline Temperatura $\left({ }^{\circ} \mathrm{C}\right)$ & 17,30 & 22,70 & 28,40 & 1,20 \\
\hline $\mathrm{pH}$ & 5,92 & 6,37 & 6,82 & 0,09 \\
\hline Alcalinidade $\left(\mathrm{mE} . \mathrm{L}^{-1}\right)$ & 0,08 & 0,22 & 0,47 & 0,04 \\
\hline Condutividade $\left(\mu \mathrm{S} . \mathrm{cm}^{-1}\right)$ & 14,70 & 32,00 & 66,50 & 6,38 \\
\hline $\mathrm{OD}\left(\mathrm{mg} \cdot \mathrm{L}^{-1}\right)$ & 2,55 & 6,07 & 8,03 & 0,57 \\
\hline $\mathrm{NO}_{3}\left(\mu \mathrm{gN} . \mathrm{L}^{-1}\right)$ & 5,12 & 28,87 & 61,88 & 6,38 \\
\hline $\mathrm{NH}_{4}\left(\mu \mathrm{g} \cdot \mathrm{L}^{-1}\right)$ & 8,30 & 39,70 & 106,02 & 10,89 \\
\hline $\mathrm{NT}\left(\mu \mathrm{g} . \mathrm{L}^{-1}\right)$ & 31,55 & 161,90 & 271,36 & 23,53 \\
\hline PTD $\left(\mu g\right.$ P.L $\left.{ }^{-1}\right)$ & 4,07 & 7,59 & 11,11 & 0,67 \\
\hline PT $\left(\mu \mathrm{g} . \mathrm{L}^{-1}\right)$ & 18,03 & 34,80 & 64,67 & 4,68 \\
\hline $\mathrm{SiH}_{4} \mathrm{O}_{4}\left(\mathrm{mgSi} . \mathrm{L}^{-1}\right)$ & 2,13 & 2,59 & 3,71 & 0,13 \\
\hline $\operatorname{MIS}\left(\mathrm{mg} \cdot \mathrm{L}^{-1}\right)$ & 1,83 & 6,89 & 18,50 & 1,74 \\
\hline $\operatorname{MOS}\left(\mathrm{mg} \cdot \mathrm{L}^{-1}\right)$ & 5,34 & 7,64 & 10,29 & 0,57 \\
\hline NT/PT (razão atômica) & 2,30 & 12,46 & 29,80 & 2,63 \\
\hline
\end{tabular}

conjunta dos dados nos dois primeiros eixos (tabela 4, figura 4). No componente 1 (48\%), houve tendência de separação das comunidades principalmente em função dos períodos hidrológicos (enchente/cheia, vazante/ seca), mas também pelo gradiente espacial. Os períodos de cheia e enchente, particularmente na região litorânea, estiveram associados aos maiores valores de densidade fitoplanctônica, clorofila- $a$, densidade das classes Chrysophyceae, Euglenophyceae, Cryptophyceae, Chloromonadophyceae, Chlorophyceae e Xanthophyceae. O componente 2 (22\%) discriminou as comunidades da região litoral nas épocas de vazante e seca pelas maiores densidades de Bacillariophyceae, Zygnemaphyceae e Oedogoniophyceae. As classes Dinophyceae e Cyanophyceae não tiveram grande importância na ordenação das épocas e dos locais amostrados.

Com relação aos resultados da ACP baseada nos dados físicos, químicos e biológicos (clorofila- $a$, densidade fitoplanctônica e das classes Cryptophyceae, Euglenophyceae, Chlorophyceae, Chrysophyceae, Bacillariophyceae, Oedogoniophyceae e Zygnemaphyceae), os dois e os três primeiros componentes explicaram, respectivamente, $65 \%$ e $82 \%$ da variabilidade total dos dados (tabela 5). No componente 1 (46\%), houve a separação dos períodos enchente/cheia dos de vazante/seca. Os primeiros períodos estiveram principalmente associados aos maiores valores de temperatura, menores concentrações de nitrato, maiores níveis fluviométricos, maiores teores de $\mathrm{CO}_{2}$ livre, às maiores densidades de Euglenophyceae, Cryptophyceae e Chrysophyceae e aos maiores valores de clorofila- $a$. O segundo eixo (19\%) separou o período de enchente, pelas maiores concentrações de amônio, do da época de cheia, quando houve maior quantitdade de oxigênio dissolvido na coluna d'água. Os dois primeiros componentes formaram, conjuntamente, três grandes grupos, com base nos períodos hidrológicos (cheia, enchente e vazante/seca), bem como tenderam a separar espacialmente as comunidades das épocas de cheia, vazante e seca. O terceiro componente $(17 \%)$ contribuiu para a separação da região litoral nas épocas de vazante e seca, basicamente pelas maiores densidades de diatomáceas, Oedogoniophyceae e Zygnemaphyceae (figura 5).

Com relação ao ficoperifíton, os valores médios da biomassa fotossintética e da densidade total apresentaram tendência de aumento nos períodos de vazante e seca (tabela 3). As classes de algas mais bem representadas foram Chlorophyceae, Oedogoniophyceae e Bacillariophyceae, com predomínio da primeira sobre as demais nos quatro períodos hidrológicos (figura 6).

\section{Discussão}

A Estação Ecológica de Jataí possui grande parte de suas características naturais preservadas, verificando-se de maneira mais acentuada apenas o efeito do pulso de inundação nas lagoas marginais existentes na região. A variação hidrológica (enchente, cheia, vazante e seca) é fator determinante na estrutura e funcionamento dos sistemas, com modificações físicas, químicas e biológicas relacionadas ao aporte de materiais provenientes do Rio Mogi-Guaçu (Rocha et al. 2000). 
Tabela 3. Valores mínimos, médios e máximos da clorofila- $a$ ( $\mu \mathrm{g} \cdot \mathrm{L}^{-1}$ para fitoplâncton e $\mu \mathrm{g} \cdot \mathrm{cm}^{-2}$ para ficoperifíton) e das densidades totais da comunidade fitoplânctonica (organismos. $\mathrm{mL}^{-1}$ ) e ficoperifítica (organismos.cm ${ }^{-2}$ ) observados nos quatro locais de coleta (FL, FI, FB, FP) e nas quatro épocas de amostragem.

Table 3. Minimum, medium, and maximum values of chlorophyll- $a$ ( $\mu \mathrm{g} \cdot \mathrm{L}^{-1}$ to phytoplankton and $\mu \mathrm{g} . \mathrm{cm}^{-2}$ to phycoperiphyton) and total phytoplanktonic (organisms. $\mathrm{mL}^{-1}$ ) and phycoperiphyton (organisms.cm ${ }^{-2}$ ) densities observed at the sampling sites (FL, FI, FB, FP) during the four sampling periods.

\begin{tabular}{|c|c|c|c|c|c|c|c|c|c|}
\hline \multicolumn{2}{|c|}{ Locais de amostragem } & \multicolumn{2}{|c|}{$\begin{array}{c}\text { novembro/1996 } \\
\text { (enchente) }\end{array}$} & \multicolumn{2}{|c|}{$\begin{array}{c}\text { fevereiro/1997 } \\
\text { (cheia) }\end{array}$} & \multicolumn{2}{|c|}{$\begin{array}{l}\text { abril/1997 } \\
\text { (vazante) }\end{array}$} & \multicolumn{2}{|c|}{$\begin{array}{l}\text { agosto/1997 } \\
\text { (seca) }\end{array}$} \\
\hline & & Clorofila- $a$ & $\begin{array}{l}\text { Densidade } \\
\text { total }\end{array}$ & Clorofila- $a$ & $\begin{array}{l}\text { Densidade } \\
\text { total }\end{array}$ & Clorofila- $a$ & $\begin{array}{l}\text { Densidade } \\
\text { total }\end{array}$ & Clorofila- $a$ & $\begin{array}{c}\text { Densidade } \\
\text { total }\end{array}$ \\
\hline \multirow[t]{3}{*}{$\mathrm{FL}$} & Valor mínimo & 5,46 & 983 & 12,01 & 2662 & 1,82 & 741 & 1,36 & 321 \\
\hline & Valor médio & 6,14 & 1388 & 12,29 & 2752 & 1,82 & 810 & 1,36 & 368 \\
\hline & Valor máximo & 6,82 & 1793 & 12,56 & 2843 & 1,82 & 879 & 1,36 & 715 \\
\hline \multirow[t]{3}{*}{ FI } & Valor mínimo & 9,55 & 2101 & 15,29 & 2502 & 3,64 & 1367 & 0,91 & 638 \\
\hline & Valor médio & 12,29 & 2273 & 15,29 & 2936 & 3,64 & 1733 & 0,91 & 1045 \\
\hline & Valor máximo & 15,01 & 2445 & 15,29 & 3370 & 3,64 & 2100 & 0,91 & 841 \\
\hline \multirow[t]{3}{*}{ FB } & Valor mínimo & 7,29 & 3312 & 17,47 & 4844 & 6,82 & 2831 & 6,37 & 2908 \\
\hline & Valor médio & 9,78 & 3802 & 17,47 & 5456 & 8,19 & 3022 & 7,96 & 3036 \\
\hline & Valor máximo & 12,29 & 4293 & 17,47 & 6069 & 7,51 & 3214 & 9,56 & 3164 \\
\hline \multirow[t]{3}{*}{ FP } & Valor mínimo & 0,13 & 179588 & 0,29 & 86149 & 0,54 & 184196 & 0,42 & 115199 \\
\hline & Valor médio & 0,32 & 205079 & 0,32 & 100063 & 0,56 & 223193 & 0,80 & 238495 \\
\hline & Valor máximo & 0,52 & 230571 & 0,36 & 113977 & 0,58 & 262190 & 1,18 & 361791 \\
\hline
\end{tabular}

No presente estudo, a variação da biomassa e a dinâmica das classes fitoplanctônicas estiveram fortemente relacionadas ao ciclo hidrológico, havendo distinção entre épocas chuvosa (enchente e cheia) e seca (vazante e seca). Considerando a comunidade fitoplanctônica como um todo, os teores mais elevados de clorofila- $a$ e as maiores densidades ocorreram nos períodos de enchente e cheia, independentemente da escala espacial. Da mesma forma, Peres \& Senna (2000) e Dias Júnior (1990) verificaram aumento da densidade total fitoplanctônica durante a estação chuvosa, atribuindo esse fenômeno ao aporte de material da planície inundada. García-de-Emiliani (1993) observou tendências semelhantes às do presente trabalho quanto à flutuação temporal da comunidade planctônica em um lago do médio Rio Paraná, constatando a importância dos pulsos de inundação nessa variação. Todavia, comportamento oposto foi verificado por Huszar (1994) nos lagos Batata e Mussurá (bacia Amazônica), nos quais as maiores densidades ocorreram no período de águas baixas.

Nos períodos de vazante e seca na Lagoa do Diogo, a comunidade fitoplanctônica apresentou tendência oposta à da época chuvosa. Ainda, nestes períodos, independentemente da escala espacial, houve distribuição mais eqüitativa entre os diversos grupos de algas, podendo estar relacionada às condições de maior disponibilidade de nutrientes, conforme evidenciado pelas razões atômicas NT/PT, que estiveram dentro da faixa considerada ideal de oferta destes elementos para as algas (10-20, segundo Reynolds 1984). Além deste fator, o menor regime de perturbações nessa época pode ter propiciado o desenvolvimento de vários grupos de algas em detrimento dos exclusivamente oportunistas.

A ACP realizada unicamente com os dados biológicos da comunidade fitoplanctônica indicou a importância de ambas as escalas, temporal e espacial, na separação das comunidades, mas principalmente do ciclo hidrológico. A mesma análise, com a inclusão das variáveis físicas e químicas ambientais, confirmou a separação das duas maiores fases hidrológicas: cheia/ enchente e vazante/seca. Verificou-se a separação das épocas de seca e de vazante unicamente para a região litoral, indicando a influência do gradiente espacial na dinâmica do sistema. Tal separação esteve relacionada, fundamentalmente, às maiores densidades de Bacillariophyceae, Oedogoniophyceae e Zygnemaphyceae no período de seca.

A densidade dos fitoflagelados (classes Euglenophyceae, Cryptophyceae e Chrysophyceae) foi um dos principais fatores que contribuiu para a separação dos grandes períodos hidrológicos na análise 
estatística, estando associada à época chuvosa. Os fitoflagelados apresentam picos de desenvolvimento após períodos de perturbação (mistura da coluna d'água pelo vento ou períodos de precipitação), quando adquirem vantagem competitiva sobre outros grupos de algas sendo, dessa forma, considerados oportunistas (Sandgren 1988, Klaveness 1988). Entretanto, outros fatores podem ter contribuído para a importância das
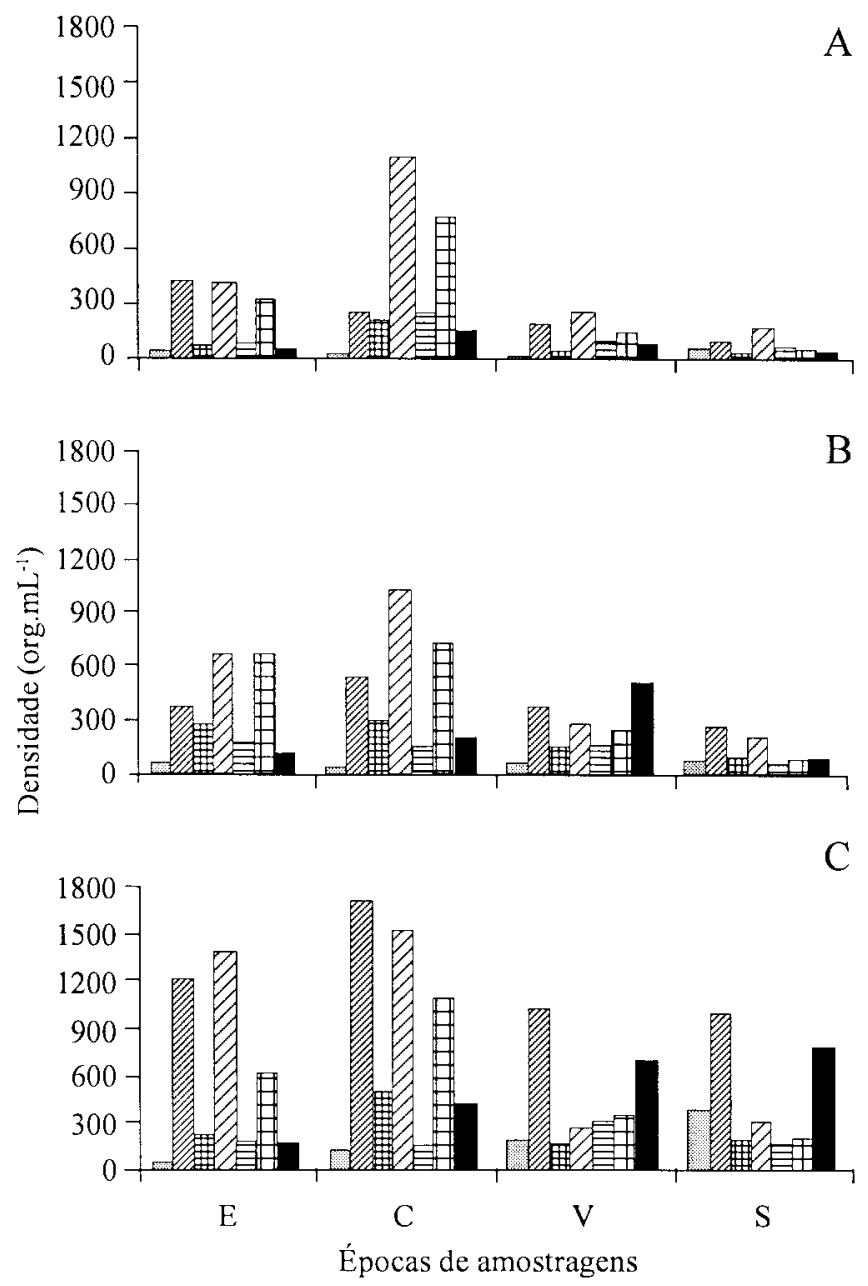

Figura 2. Variação temporal das densidades (organismos. $\mathrm{mL}^{-1}$ ) das classes fitoplanctônicas nos locais de coleta FL (A), FI (B) e FB (C) nas quatro épocas de amostragem ( $\mathrm{E}=$ enchente, $\mathrm{C}=$ cheia, $\mathrm{V}=$ vazante, $\mathrm{S}=$ seca). $=$ Bacillariophyceae, $\square=$ Chlorophyceae, $\mathbb{}=$ Chrysophyceae, $\square=$ Cryptophyceae, 日-Cyanophyceae, $甘=$ Euglenophyceae, $\mathbf{m}=$ Outros grupos.

Figure 2. Temporal variation of phytoplankton classes densities (organisms. $\mathrm{mL}^{-1}$ ) in the sampling sites FL (A), FI (B) and $\mathrm{FB}(\mathrm{C})$ during the four sampling periods $(\mathrm{E}=$ high water,

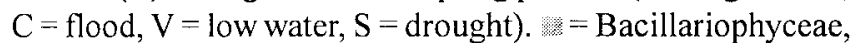

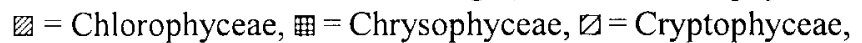
目=Cyanophyceae, $\boxplus=$ Euglenophyceae, $\boldsymbol{m}=$ Others groups.

$\mathrm{C}$ classes de algas mencionadas na ordenação dos períodos, como as maiores concentrações médias de matéria orgânica e maiores teores de fósforo total, favorecendo as Euglenophyceae e as Chrysophyceae, respectivamente.

Diferentemente, Peres \& Senna (2000) observaram que a ocorrência da classe Euglenophyceae esteve associada com o período seco e que Chrysophyceae
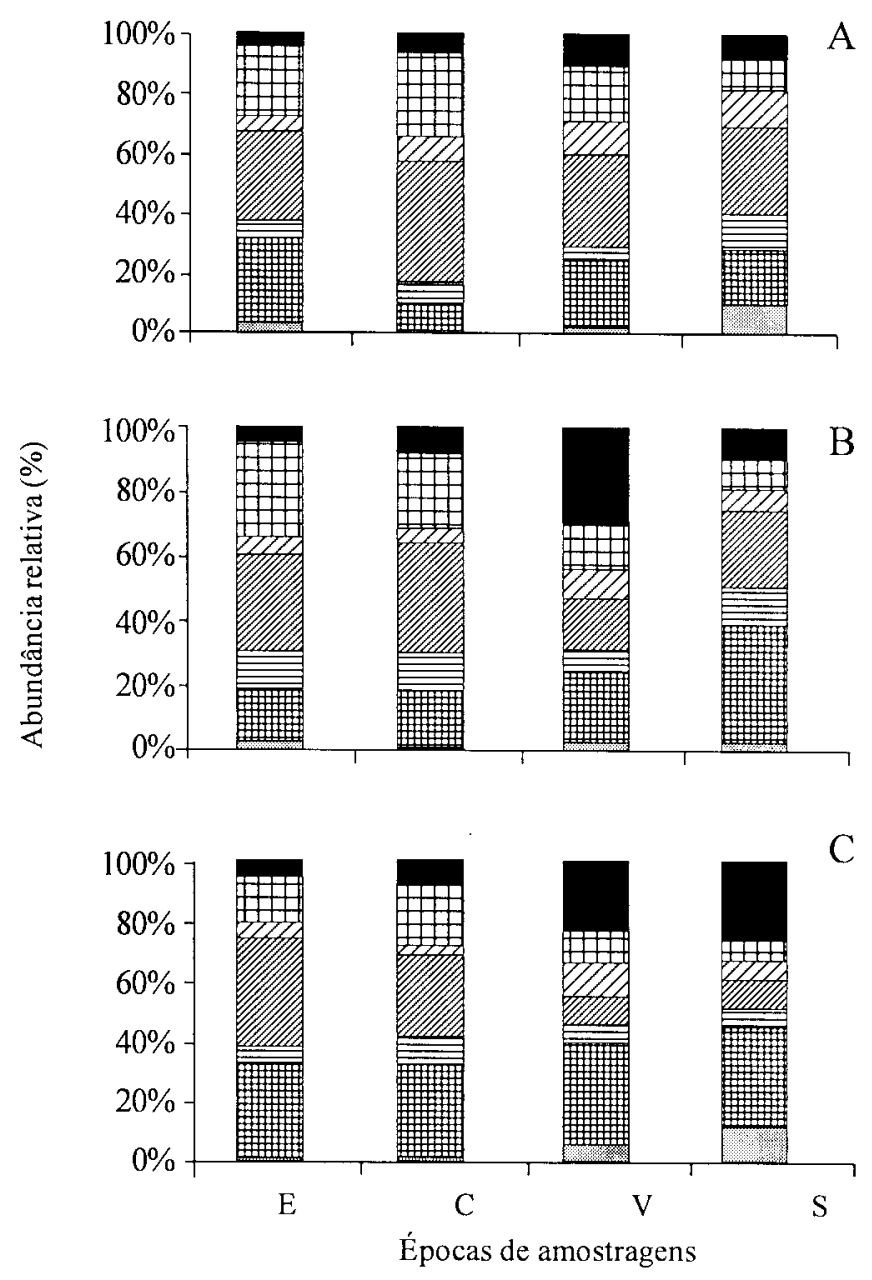

Figura 3. Variação temporal da abundância relativa (\%) das classes fitoplanctônicas nos locais de coleta FL (A), FI (B) e $\mathrm{FB}(\mathrm{C})$ nas quatro épocas de amostragem $(\mathrm{E}=$ enchente, $\mathrm{C}=$ cheia, $\mathrm{V}=$ vazante, $\mathrm{S}=$ seca $) . \quad=$ = Bacillariophyceae, 困=Chlorophyceae, 目=Chrysophyceae, 盾=Cryptophyceae, $\nabla=$ Cyanophyceae, $\boxplus$ = Euglenophyceae, $\mathbf{m}=$ Outros grupos.

Figure 3. Temporal variation of relative abundance $(\%)$ of phytoplankton classes in the sampling sites FL (A), FI (B) and $F B(C)$ during the four sampling periods $(E=$ high water, $\mathrm{C}=$ flood, $\mathrm{V}=$ low water, $\mathrm{S}=$ drought $) . \mathrm{m}=$ Bacillariophyceae, 田=Chlorophyceae, 目=Chrysophyceae, $\nabla=$ Cyanophyceae, $\mathbb{\theta}=$ Euglenophyceae, $\boldsymbol{\square}=$ Others groups. 
não apresentou relação alguma com as fases do ciclo hidrológico. Por outro lado, Dias Júnior (1990) verificou que Chrysophyceae esteve associada principalmente à época seca.

É importante salientar que a classe Chlorophyceae foi uma das mais bem representadas em todos os períodos e locais de amostragem e, portanto, não sendo fator discriminante para separação das épocas de

Tabela 4. Correlação da biomassa e densidades do fitoplâncton com os componentes da ACP 1 e 2.

Table 4. Correlation of phytoplankton biomass and densities to PCA components 1 and 2.

\begin{tabular}{lcc}
\hline Variáveis & Componente 1 & Componente 2 \\
\hline Clorofila- $a$ & $0,907^{*}$ & 0,051 \\
Fitoplâncton (DT) & $0,956^{*}$ & $-0,215$ \\
Bacillariophyceae & 0,190 & $-0,915^{*}$ \\
Chloromonadophyceae & $0,823^{*}$ & 0,263 \\
Chlorophyceae & $0,802^{*}$ & $-0,375$ \\
Chrysophyceae & $0,916^{*}$ & 0,039 \\
Cryptophyceae & $0,868^{*}$ & 0,300 \\
Cyanophyceae & 0,376 & $-0,411$ \\
Dinophyceae & $-0,203$ & $-0,276$ \\
Euglenophyceae & $0,902^{*}$ & 0,309 \\
Oedogoniophyceae & $-0,021$ & $-0,825^{*}$ \\
Xanthophyceae & $0,744^{*}$ & $-0,080$ \\
Zygnemaphyceae & 0,020 & $-0,836^{*}$ \\
\% Total da Variância & 47,88 & 22,42 \\
\% Acumulada da Variância & 47,88 & 70,30 \\
\hline
\end{tabular}

* valores de maior correlação com os eixos

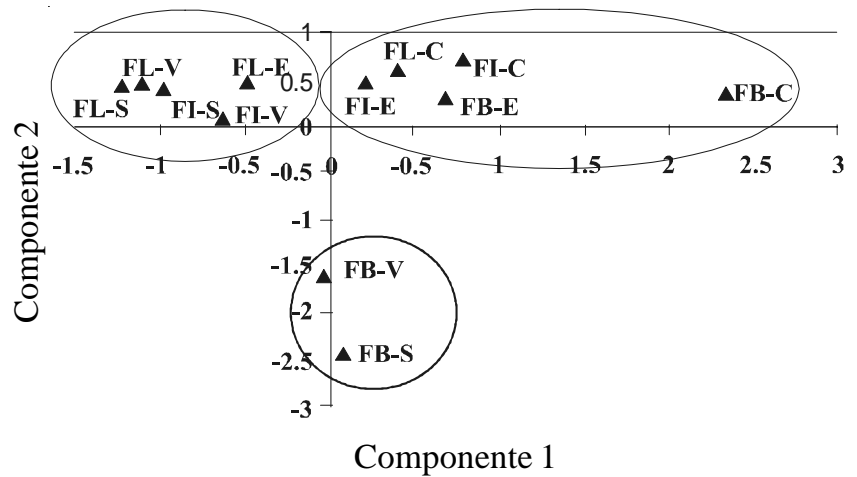

Figura 4. Ordenação pela ACP dos locais (FL, FI, FB) e das épocas de amostragem em função das variáveis do fitoplâncton $(\mathrm{E}=$ enchente, $\mathrm{C}=$ cheia, $\mathrm{V}=$ vazante, $\mathrm{S}=$ seca).

Figure 4. PCA ordenation of sites (FL, FI, FB) and sampling periods based on phytoplankton $(\mathrm{E}=$ high water, $\mathrm{C}=$ flood, $\mathrm{V}=$ low water, $\mathrm{S}=$ drought).
Tabela 5. Correlação das variáveis físicas, químicas e do fitoplâncton com os componentes 1,2 e 3 da ACP.

Table 5. Correlation of physical, chemical and phytoplankton variables to PCA components 1, 2 and 3 .

\begin{tabular}{|c|c|c|c|}
\hline \multirow[t]{2}{*}{ Variáveis } & \multirow{2}{*}{$\begin{array}{c}\text { Componente } \\
1\end{array}$} & \multirow{2}{*}{$\begin{array}{c}\text { Componente } \\
2\end{array}$} & \multirow{2}{*}{$\begin{array}{c}\text { Componente } \\
3\end{array}$} \\
\hline & & & \\
\hline $\begin{array}{l}\text { Nível Hidrométrico } \\
\text { do Rio Mogi-Guaçu }\end{array}$ & $\mathrm{u}^{-0,883^{*}}$ & 0,095 & $-0,378$ \\
\hline Temperatura & $-0,932^{*}$ & 0,128 & $-0,280$ \\
\hline Transparência & 0,530 & 0,208 & $-0,227$ \\
\hline $\mathrm{pH}$ & $-0,809^{*}$ & $-0,404$ & 0,100 \\
\hline $\mathrm{CO}_{2}$ livre & $-0,859^{*}$ & $-0,329$ & $-0,210$ \\
\hline Condutividade & $-0,809^{*}$ & $-0,573$ & 0,094 \\
\hline OD & 0,528 & $0,762^{*}$ & 0,001 \\
\hline $\mathrm{NO}_{3}$ & $0,884^{*}$ & $-0,127$ & 0,350 \\
\hline $\mathrm{NH}_{4}$ & $-0,498$ & $-0,776^{*}$ & 0,337 \\
\hline NT & 0,576 & $-0,469$ & 0,602 \\
\hline PTD & $-0,669$ & $-0,070$ & 0,251 \\
\hline PT & $-0,694$ & $-0,585$ & 0,274 \\
\hline $\mathrm{SiH}_{4} \mathrm{O}_{4}$ & $-0,491$ & 0,298 & 0,419 \\
\hline MIS & $-0,525$ & $-0,628$ & 0,548 \\
\hline MOS & $-0,632$ & 0,157 & 0,608 \\
\hline Clorofila-a & $-0,823^{*}$ & 0,471 & $-0,006$ \\
\hline Fitoplâncton (DT) & $-0,696$ & 0,624 & 0,027 \\
\hline Bacillariophyceae & 0,144 & 0,384 & $0,860^{*}$ \\
\hline Chlorophyceae & $-0,504$ & 0,539 & 0,479 \\
\hline Chrysophyceae & $-0,756^{*}$ & 0,480 & 0,054 \\
\hline Cryptophyceae & $-0,831^{*}$ & 0,359 & $-0,116$ \\
\hline Euglenophyceae & $-0,848^{*}$ & 0,408 & $-0,227$ \\
\hline Oedogoniophyceae & 0,258 & 0,258 & 0,604 \\
\hline Zygnemaphyceae & 0,184 & 0,296 & $0,787^{*}$ \\
\hline \% total da Variância & 45,75 & 19,46 & 16,71 \\
\hline $\begin{array}{l}\text { \% acumulada da } \\
\text { Variância }\end{array}$ & 45,75 & 65,21 & 81,92 \\
\hline
\end{tabular}

* valores de maior correlação com os eixos

amostragem, corroborando, assim, os resultados de Peres \& Senna (2000).

Considerando a escala espacial, os maiores valores de clorofila- $a$ e de densidade fitoplanctônica foram encontrados na região litoral. Particularmente, as Chlorophyceae apresentaram maiores densidades nessa região, diminuindo em abundância relativa (\%) em direção à água aberta. As maiores densidades de clorofíceas dentro do estande de macrófitas podem estar relacionada à maior disponibilidade de nutrientes (fósforo total dissolvido e fósforo total) na região litorânea. No entanto, este grupo de algas possui vários representantes de hábitat planctônico e também perifítico. Sendo assim, deve-se considerar o aporte de algas para a região litoral 
A
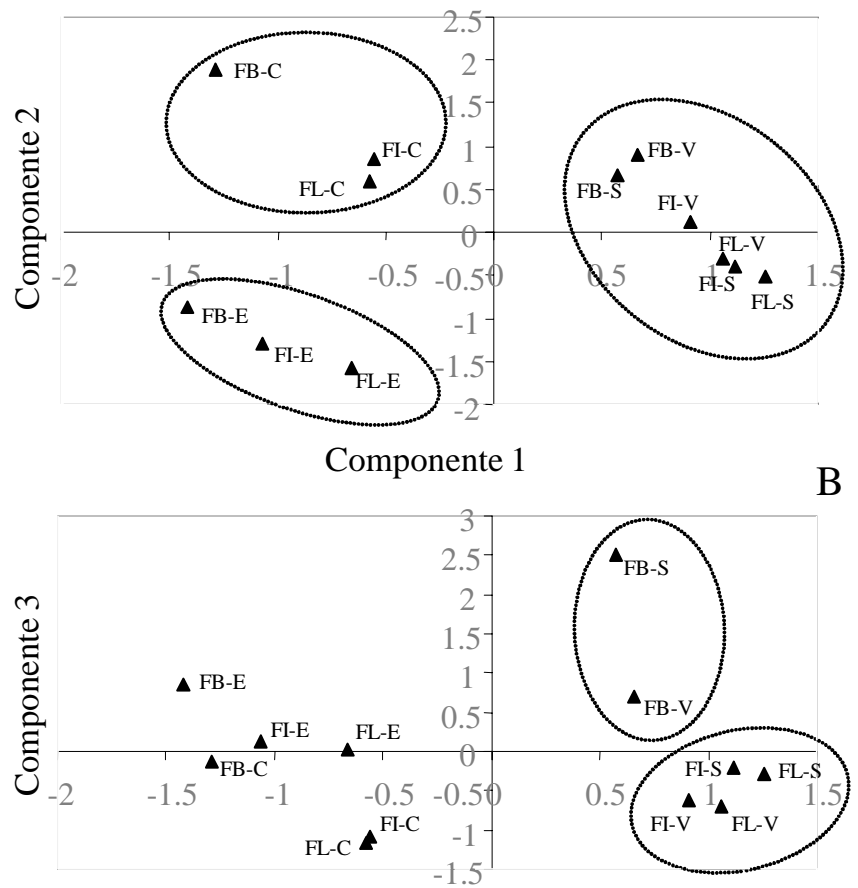

Componente 1

Figura 5. Ordenação pela ACP dos locais (FL, FI, FB) e das épocas de amostragem em função das variáveis físicas, químicas e do fitoplâncton. $\mathrm{A}=$ componentes $1 \times 2$; $\mathrm{B}=$ componentes $1 \times 3 .(\mathrm{E}=$ enchente, $\mathrm{C}=$ cheia, $\mathrm{V}=$ vazante, $\mathrm{S}=$ seca)

Figure 5. PCA ordination of sites (FL, FI, FB) and sampling periods in relation to chemical, physical and phytoplankton variables. $\mathrm{A}=$ components $1 \times 2$; $\mathrm{B}=$ components $1 \times 3$. $(\mathrm{E}=$ high water, $\mathrm{C}=$ flood, $\mathrm{V}=$ low water, $\mathrm{S}=$ drought).

via perifíton, principalmente em época chuvosa. Tal hipótese pode ser reforçada pelos resultados encontrados para a comunidade perifítica, na qual houve dominância da classe Chlorophyceae durante o período de seca/ vazante e queda durante a cheia. Provavelmente, a ação mecânica e a turbulência da água, causadas pelos distúrbios dos pulsos de inundação e pela chuva concorreram para o desprendimento dos organismos perifíticos. Dessa forma, na época de cheia, houve pico de clorofíceas no "plâncton" da região litoral (FB), mas não em água aberta (FL). Rodrigues \& Bicudo (2004) demonstraram claramente que as perturbações (variações acentuadas do nível hidrológico do Rio Paraná e fortes ventos) foram os principais fatores controladores da sucessão perifítica em substrato artificial em intervalos curtos de tempo. Observou mudanças evidentes no curso do processo sucessional, nas taxas de produção e de exportação de biomassa e nas classes de algas dominantes na fase de águas altas em ambientes lêntico e semilótico na planície de inundação do alto rio Paraná. Além disso, conforme afirmado por Happey-Wood (1988), muitas clorofíceas planctônicas possuem o desenvolvimento derivado do hábitat perifítico, ou seja, tendo como inóculo a comunidade perifítica.

$\mathrm{Na}$ ACP baseada apenas nas variáveis biológicas, pode-se notar que a comunidade planctônica da região litoral se distanciou das demais. Durante a cheia, caracterizou-se principalmente pela maior biomassa e pelos valores mais elevados de densidades total e de várias classes (Chrysophyceae, Euglenophyceae, Cryptophyceae e Chlorophyceae), enquanto na seca e vazante, pelas maiores densidades de Bacillariophyceae, Zygnemaphyceae e Oedogoniophyceae. Mesmo considerando a ACP feita a partir de todas as variáveis,
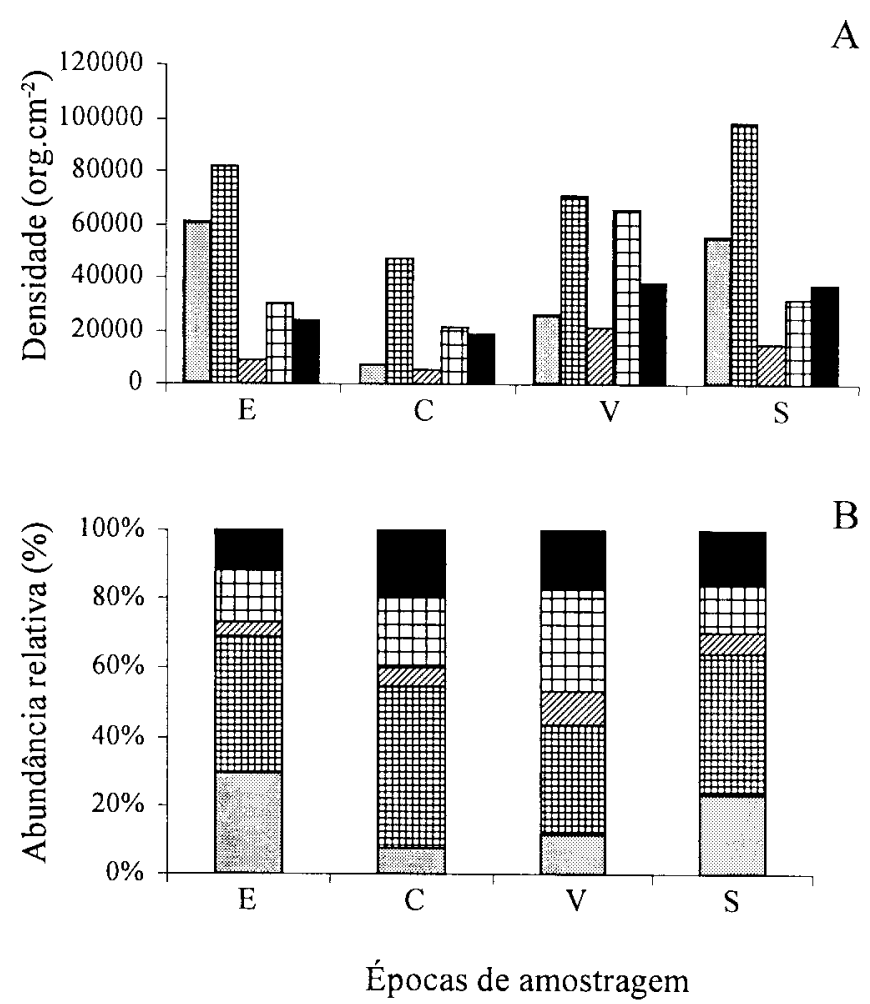

Figura 6. Variação temporal da densidade (organismos. $\mathrm{cm}^{-2}$ ) (A) e da abundância relativa (\%) (B) das classes ficoperifíticas nos quatro períodos de amostragem $(\mathrm{E}=$ enchente, $\mathrm{C}=$ cheia, $\mathrm{V}=$ vazante, $\mathrm{S}=$ seca). : $=$ Bacillariophyceae, $\mathbf{t}=$ Chlorophyceae, $\Xi=$ Cyanophyceae, $\boxminus=$ Oedogoniophyceae, $\mathbf{\square}=$ Outros grupos.

Figure 6. Temporal variation of phycoperiphyton classes densities (organisms. $\mathrm{cm}^{-2}$ ) (A) and relative abundance (\%) (B) during the four sampling periods $(\mathrm{E}=$ high water, $\mathrm{C}=$ flood, $\mathrm{V}=$ low water, $\mathrm{S}=$ drought). $\boldsymbol{\nabla}=$ Cyanophyceae, $\mathbf{B}=$ Oedogoniophyceae, $\boldsymbol{\square}=$ Outros grupos. 
verificou-se que principalmente na cheia, na vazante e na seca a região litoral se diferenciou das demais regiões.

Embora a escala espacial não tenha sido a maior fonte de variabilidade da comunidade fitoplanctônica no sistema estudado no gradiente litoral-limnético, a região litoral foi a que apresentou características mais específicas.

A comunidade ficoperifítica apresentou tendências bem distintas da fitoplanctônica. Além de apresentar os maiores valores de clorofila- $a$ e densidade total nos períodos de vazante/seca, teve maior representatividade das classes Chlorophyceae, Oedogoniophyceae e Bacillariophyceae. Durante a cheia, as densidades total e dos principais grupos de algas sofreram redução, muito provavelmente relacionada aos pulsos de inundação, como discutido anteriormente.

Apesar da diferença das unidades de medidas dos atributos das comunidades planctônica e perifítica, é possível inferir que os elevados valores de densidade e de clorofila- $a$ na região litoral (plâncton e perifíton) devem-se, principalmente, à presença das macrófitas aquáticas, que fornecem importante substrato para o desenvolvimento de algas, bem como à maior concentração de nutrientes (ortossilicato, fósforo total e fósforo total dissolvido) e temperatura nessa região. A zona litoral, como enfatizado por Wetzel (1990, 1996), é uma área de grande disponibilidade e intensa ciclagem de nutrientes. Ainda, segundo Wetzel (1990, 1996), o ortofosfato é intensamente reciclado nessa região, sendo pouco translocado para água aberta, o que certamente explica as concentrações sempre abaixo do limite de detecção do método. Confer (1972), através de quantificações do fluxo de fósforo entre fitoplâncton e perifíton, verificou que o perifíton (particularmente as formas verdes filamentosas) assimila rapidamente grandes quantidades de fósforo, reduzindo o conteúdo desse nutriente na coluna d'água.

Provavelmente, as elevadas densidades de diatomáceas no perifíton estiveram relacionadas principalmente ao seu tipo de hábitat (Willén 1991, Round et al. 1990). Os resultados obtidos na ACP para a comunidade fitoplanctônica dão suporte a esta afirmativa, já que as diatomáceas estiveram mais associadas à escala espacial (região litoral) do que à temporal. Assim como para as diatomáceas, o tipo de hábitat deve ter contribuído para a boa representação das Oedogoniophyceae, que compreendem formas filamentosas com estruturas muito eficientes de fixação (Oedogonium, Bulbochaete). Para a Lagoa do Infernão, Schwarzbold (1992) verificou que a classe Oedogoniophyceae foi a principal representante do ficoperifíton, tanto em porcentagem quanto em biovolume nos períodos de cheia e de seca, estando as classes Chlorophyceae e Bacillariophyceae presentes em baixas porcentagens e biovolumes.

Na Lagoa do Diogo, o ficoperifíton parece não sofrer grandes alterações no período de chuvas com relação à proporção dos diversos grupos de algas. Sand-Jensen (1983) considera que, nas fases iniciais de colonização de um substrato, a matriz perifítica, por ser pouco densa, reflete melhor as condições ambientais circundantes, enquanto que com o desenvolvimento os processos internos da comunidade tornam-se mais importantes. Como o material foi coletado a partir de pecíolos de E. azurea em estádio adulto, a comunidade perifítica, muito provavelmente, já se encontrava bem desenvolvida e, assim, mais independente das mudanças físicas e químicas durante o ciclo hidrológico.

Comparando-se as classes de algas das comunidades planctônicas nos três locais de amostragem e da comunidade perifítica, o fitoplâncton sofreu maiores alterações em sua composição do que o ficoperifíton durante as épocas de enchente e cheia. Todavia, em relação à densidade, observou-se diminuição na densidade da comunidade perifítica, com o respectivo aumento do fitoplâncton da região litoral, principalmente de Bacillariophyceae, Oedogoniophyceae, Chlorophyceae e Zygnemaphyceae.

Os resultados do presente estudo evidenciam o importante papel do regime hidrológico sobre as flutuações das comunidades planctônicas e perifíticas, bem como sobre a interação e trocas entre ambas na Lagoa do Diogo. Quanto ao último aspecto, pode-se dizer que a exportação do perifíton para outros hábitats pode ter importantes implicações para a dinâmica e o funcionamento da comunidade fitoplanctônica da região litorânea.

Como enfatizado por Goldsborough \& Robinson (1996), em ambientes rasos, como em áreas alagadas de modo geral, é difícil estabelecer limites de hábitat para as populações algais, uma vez que há grande interação entre os distintos compartimentos do sistema. Essa interação é influenciada principalmente pela hidrologia, estabilidade da coluna da água, disponibilidade de superfícies colonizáveis, entre outros fatores.

Especialmente em sistemas rasos, os estudos sobre interação perifíton/plâncton merecem maior atenção, uma vez que podem trazer importantes elucidações sobre os padrões de flutuação da estrutura e do funcionamento das comunidades de algas e, conseqüentemente, sobre o metabolismo e fluxos de energia nesses ecossistemas aquáticos. 
Agradecimentos - À coordenação do Programa de Pós-graduação em Ecologia e Recursos Naturais da Universidade Federal de São Carlos, pelo apoio logístico e à Capes (Coordenadoria de Aperfeiçoamento de Pessoal de Nível Superior) pela bolsa de mestrado concedida ao primeiro autor.

\section{Referências bibliográficas}

BARBOSA, F.A.R., BICUDO, C.E.M. \& HUSZAR, V.M. 1995. Phytoplankton studies in Brazil: community structure variation and diversity. In Limnology in Brazil (J.G. Tundisi, C.E.M. Bicudo \& T. Matsumura-Tundisi, eds.). Academia Brasileira de Ciências e Sociedade Brasileira de Limnologia, Rio de Janeiro, p.19-36.

BICUDO, D.C. 1990. Considerações sobre metodologias de contagem de algas do perifíton. Acta Limnologica Brasiliensia 3:459-475.

BICUDO, D.C., NECCHI JÚNIOR, O. \& CHAMIXAES, C.B. 1995. Periphyton studies in Brazil: present status and perspectives. In Limnology in Brazil (J.G. Tundisi, C.E.M. Bicudo \& T. Matsumura-Tundisi, eds.). Academia Brasileira de Ciências e Sociedade Brasileira de Limnologia, Rio de Janeiro, p.37-42.

BONETTO, C.A., ZALOCAR, Y. \& LANCELLE, H.G. 1984. A limnological study of an oxbow-lake covered by Eichhornia crassipes in the Paraná River. Internationale Vereinigung für Theoretische und Angewandte Limnologie 22:1315-1318.

CONFER, J.L. 1972. Interrelations among phytoplankton attached algae, and the phosphorus cycle in artificial open systems. Ecological Monographs 42:1-23.

DIAS JÚNIOR, C. 1990. Ciclo anual do fitoplâncton e algumas variáveis ambientais na Lagoa do Infernão (SP). Dissertação de mestrado, Universidade Federal de São Carlos, São Carlos.

GARCÍA-DE-EMILIANI, M.O. 1993. Seasonal succession of phytoplankton in a lake of the Paraná river floodplain, Argentina. Hydrobiologia 264:101-114.

GOLDSBOROUGH, L.G. \& ROBINSON, G.G. 1996. Pattern in wetlands. In Algal ecology: freshwater benthic ecossystems (R.J. Stevenson, M.L. Bothwell \& R.L. Lowe, eds.). Academic Press, San Diego, p.77-117.

GOLTERMAN, H.L., CLYMO, R.S. \& OHNSTAD, M.A.M. 1978. Methods for physical and chemical analysis of freshwaters. Blackwell Scientific Publications, Oxford.

HAPPEY-WOOD, C.M. 1988. Ecology of freshwater planktonic green algae. In Growth and reproductive strategies of freshwater phytoplankton (C.D. Sandgren, ed.). Cambridge University Press, Cambridge, p.175-226.

HUSZAR, V.L.M. 1994. Fitoplâncton de um lago amazônico impactado por rejeito de bauxita (Lago Batata, Pará, Brasil): estrutura da comunidade, flutuações espaciais e temporais. Tese de doutorado, Universidade Federal de São Carlos, São Carlos.
KLAVENESS, D. 1988. Ecology of the Cryptomonadida: a first review. In Growth and reproductive strategies of freshwater phytoplankton (C.D. Sandgren, ed.). Cambridge University Press, Cambridge, p.105-133.

KOROLEFF, F. 1976. Determination of nutrients. In Methods of seawater analysis (K. Grasshoff, ed.). Verlag Chemie Weinheim, New York, p.117-181.

KRUSCHE, A.V. 1989. Caracterização biogeoquímica da Lagoa do Diogo, uma lagoa marginal do Rio Mogi-Guaçu (Estação Ecológica de Jataí, Luiz Antonio, SP). Dissertação de mestrado, Universidade Federal de São Carlos, São Carlos.

MACKERETH, F.J.H., HERON, J. \& TALLING J.F. 1978. Water analysis: some revised methods for limnologists. Freshwater Biological Association Scientific Publication 36, Ambleside.

MAGRIN, A.G.E. 1998. Estrutura e dinâmica de diatomáceas no plâncton e no perifíton (epifíticas + metafíticas) da Lagoa do Diogo, Estação Ecológica de Jataí, município de Luiz Antônio, São Paulo: uma lagoa permanentemente ligada ao Rio Mogi-Guaçu. Tese de doutorado, Universidade Federal de São Carlos, São Carlos.

MAGRIN, A.G.E. \& SENNA, P.A.C. 1997. Composição e dinâmica de diatomáceas planctônicas em uma lagoa da planície de inundação do médio Mogi-Guaçu, estado de São Paulo, Brasil. Anais do VIII Seminário Regional de Ecologia 8:247-276.

MANLY, B.F.J. 1986. Multivariate statistical methods: a primer. Chapman, New York.

MARGALEF, R. 1983. Limnologia. Omega, Barcelona.

NUSCH, E.A. \& PALME, G. 1975. Biologische methoden für die praxis der gewässeruntersuchung. Bestimmung des Chlorophyll a und phaeopigmentgehaltes in oberflächenwasser. GWF-Wasser/Abwasser 116:562-565.

PERES, A.C. \& SENNA, P.A.C. 2000. Estudo quantitativo e estatístico do fitoplâncton da lagoa do Diogo em um ciclo hidrológico (1995-1996). In Estação Ecológica de Jataí (J.E. Santos \& J.S.R. Pires, eds.). RiMa, São Carlos, v.2, p.483-495.

PIRES, A.M.Z.C.R., SANTOS, J.E. \& PIRES, J.S.R. 2000. Caracterização e diagnóstico ambiental de uma unidade da paisagem. Estudo de caso: Estação Ecológica de Jataí e Estação Experimental de Luiz Antônio. In Estação Ecológica de Jataí (J.E. Santos \& J.S.R. Pires, eds.). RiMa, São Carlos, v.1, p.1-25.

REYNOLDS, C.S. 1984. The ecology of freshwater phytoplankton. Cambridge University Press, Cambridge.

ROCHA, O., ESPÍNDOLA, E.G., RIETZLER, A.C. \& SANTOSWISNIEWSKI, M.J. 2000. Diversidade do zooplâncton nas lagoas marginais do Rio Mogi-Guaçu: III. Copepoda (Crustácea). In Estação Ecológica de Jataí (J.E. Santos \& J.S.R. Pires, eds.). RiMa, São Carlos, v.1, p.587-598. 
RODRIGUES, L. \& BICUDO, D.C. 2004. Periphytic algae. In The upper Paraná River and its floodplain (S.M. Thomaz, A.A. Agostinho \& N.S. Hahn, eds.). Backhuys Publishers, Leiden, p.125-143.

ROS, J. 1979. Práctica de ecología. Omega, Barcelona.

ROUND, F.E. 1965. The biology of the algae. Edward Arnold, London.

ROUND, F.E. 1971. The taxonomy of the Chlorophyta, 2. British Phycological Journal 6:235-264.

ROUND, F.E., CRAWFORD, R.M. \& MANN, D.G. 1990. The diatoms. Cambridge University Press, Cambridge.

SANDGREN, C.D. 1988. The ecology of Chrysophyte flagellates: their growth and perennation strategies as freshwater phytoplankton. In Growth and reproductive strategies of freshwater phytoplankton (C.D. Sandgren, ed.). Cambridge University Press, Cambridge, p.9-103.

SAND-JENSEN, K. 1983. Physical and chemical parameters regulating growth of periphytic communities. In Periphyton of freshwater ecosystems (R.G. Wetzel, ed.). Dr. W. Junk Publishers, The Hague, p.63-71.

SANTOS, J.E. \& PIRES, J.S.R. (eds.). 2000. Estação Ecológica de Jataí. RiMa, São Carlos, v.2, p.347-867.

SCHWARZBOLD, A. 1992. Efeitos do regime de inundação do Rio Mogi-Guaçu (SP) sobre a estrutura, diversidade, produção e estoques do perifíton da Lagoa do Infernão. Tese de doutorado, Universidade Federal de São Carlos, São Carlos.

SCHWARZBOLD, A., ESTEVES, F.A. \& PANOSSO, R.F. 1990. Relação entre peso seco e clorofila- $a$ do perifíton em função de diferentes idades e épocas de coletas de pecíolos de Eichhornia azurea Kunth. Acta Limnologica Brasiliensia 3:493-515.

SENNA, P.A.C., PERES, A.C. \& KOMÁREK, J. 1998. Coelomorum tropicalis, a new cyanoprokaryotic species from São Paulo State, Brazil. Nova Hedwigia 67:93-100.

STRICKLAND, J.D. \& PARSONS, T.R. 1960. A manual of sea water analysis. Fisheries Research Board of Canada, Ottawa ( $2^{\text {nd }}$ edition, Bulletin $\left.n^{\circ} 125\right)$.
TANIGUCHI, G.M., PERES, A.C., SENNA, P.A.C. \& BICUDO, D.C. 1998. Desmidiaceae filamentosas, Mesotaeniaceae e Gonatozygaceae de uma lagoa marginal do Rio MojiGuaçu, Estação Ecológica de Jataí, Estado de São Paulo. Hoehnea 25:149-167.

TANIGUCHI, G.M., BICUDO, D.C. \& SENNA, P.A.C. 2000. Intercâmbio populacional de desmídias planctônicas e perifíticas na Lagoa do Diogo, planície de inundação do Rio Mogi-Guaçu. In Estação Ecológica de Jataí (J.E. Santos \& J.S.R. Pires, eds.). RiMa, São Carlos, v.2, p.431-444.

TANIGUCHI, G.M., PERES, A.C., SENNA, P.A.C. \& COMPÈRE, P. 2003. The desmid genera Cosmarium, Actinotaenium, and Cosmocladium from an oxbow lake, Jataí Ecological Station (Southeastern Brazil). Systematics and Geography of Plants 73:133-159.

TEIXEIRA, C., TUNDISI, J.G. \& KUTNER, M.B. 1965. Plankton studies in a mangrove. II : The standing-stock and some ecological factors. Boletim do Instituto Oceanográfico 24: $23-41$.

UTERMÖHL, H. 1958. Zur Vervollkomnung der quantitativen phytoplankton-methodik. Internationale Vereinigung für Theoretische und Angewandte Limnologie Mitteilungen 9:1-38.

WETZEL, R.G. 1990. Land-water interfaces: metabolic and limnological regulators. Internationale Vereinigung für Theoretische und Angewandte Limnologie 24:6-24.

WETZEL, R.G. 1996. Benthic algae and nutrient cycling in lentic freshwater ecosystems. In Algal ecology: freshwater benthic ecossystems (R.J. Stevenson, M.L. Bothwell \& R.L. Lowe, eds.). Academic Press, San Diego, p.641-667.

WETZEL, R.G. \& LIKENS, G.E. 1991. Limnological analyses. Springer-Verlag, New York.

WILLÉN, E. 1991. Planktonic diatoms - an ecological review. Algological Studies 62:69-106. 